\title{
Conformational Heterogeneity in a Fully Complementary DNA Three-Way Junction with a GC-Rich Branchpoint
}

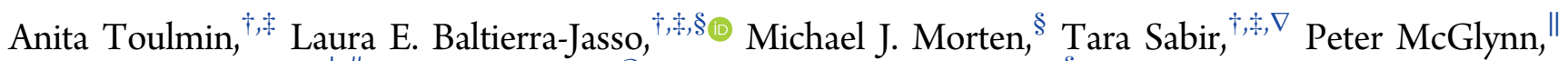 \\ Gunnar F. Schröder, ${ }^{\perp, \#}$ Brian O. Smith, ${ }^{@}$ and Steven W. Magennis, ${ }^{*, \S}$ \\ ${ }^{\dagger}$ The School of Chemistry, The University of Manchester, Oxford Road, Manchester M13 9PL, U.K. \\ ${ }^{\ddagger}$ The Photon Science Institute, The University of Manchester, Alan Turing Building, Oxford Road, Manchester M13 9PL, U.K. \\ ${ }^{\S}$ School of Chemistry, WestCHEM, University of Glasgow, Joseph Black Building, University Avenue, Glasgow G12 8QQ, U.K. \\ "Department of Biology, University of York, Wentworth Way, York YO10 5DD, U.K. \\ ${ }^{\perp}$ Institute of Complex Systems (ICS-6), Forschungszentrum Jülich, 52425 Jülich, Germany \\ ${ }^{\#}$ Physics Department, Heinrich-Heine Universität Düsseldorf, Düsseldorf, Germany \\ ${ }^{\circledR}$ Institute of Molecular, Cell and Systems Biology, University of Glasgow, Glasgow G12 8QQ U.K.
}

\section{Supporting Information}

ABSTRACT: DNA three-way junctions (3WJs) are branched structures that serve as important biological intermediates and as components in DNA nanostructures. We recently derived the global structure of a fully complementary $3 \mathrm{WJ}$ and found that it contained unpaired bases at the branchpoint, which is consistent with previous observations of branch flexibility and branchpoint reactivity. By combining high-resolution single-molecule Förster resonance energy transfer, molecular modeling, time-resolved ensemble fluorescence spectroscopy, and the first ${ }^{19} \mathrm{~F}$ nuclear magnetic resonance observations of fully complementary $3 \mathrm{WJ}$, we now show that the $3 \mathrm{WJ}$ structure can adopt multiple distinct conformations depending upon the sequence at the branchpoint. A $3 \mathrm{WJ}$ with a GC-rich branchpoint adopts an open conformation with unpaired bases at the branch and at least one additional conformation with an increased number of base interactions at the branchpoint. This structural diversity has implications for branch interactions and processing in vivo and for technological applications.

$\mathrm{B}$ ranched nucleic acid molecules are key intermediates and structural elements in vivo ${ }^{1,2}$ and are important components in structural and dynamic DNA nanoscience. ${ }^{3}$ Arguably the simplest branched structure is the three-way junction (3WJ), formed from three single nucleic acid strands. We recently used high-resolution single-molecule Förster resonance energy transfer (SM-FRET ${ }^{4}$ ) to show that a DNA 3WJ with a branchpoint that is not GC-rich does not adopt a fully paired conformation as expected but instead has a nanoscale cavity at the branchpoint..$^{5}$ The structures of fully complementary $3 \mathrm{WJ}$ have previously eluded structural characterization at atomic resolution because their dynamic nature has precluded their crystallization and rendered ${ }^{1} \mathrm{H}$ nuclear magnetic resonance (NMR) signals of the junction bases unobservable. Since then, 3WJs have continued to attract attention in a range of contexts, including for controlled charge transport, ${ }^{6-8}$ ion sensing, molecular recognition, ${ }^{10}$ and as scaffolds for light harvesting. $^{11,12}$

In this work, we examine whether the expanded branchpoint described previously ${ }^{5}$ is a general phenomenon for all fully complementary $3 \mathrm{WJ}$ s or if it is dependent on sequence. We use SM-FRET and molecular dynamics (MD) simulations to study local and global 3WJ structure. In addition, we report the first use of ${ }^{19} \mathrm{~F}$ NMR spectroscopy to study fully complementary $3 \mathrm{WJ}$ s, by incorporating a 5-fluorocytosine near the branchpoint. We find that a $3 \mathrm{WJ}$ with a GC-rich branchpoint can adopt at least two conformations in solution. The major conformation is similar to that previously observed, with unpaired bases at the branch and a Y-shaped, pyramidal structure. There is also at least one minor conformation with an altered branchpoint structure, which we attribute to an increased level of base pairing at the branch. These structures are in exchange on the supramillisecond time scale. The structural heterogeneity observed upon changing the branch sequence will have consequences for the reactivity of fully complementary $3 \mathrm{WJ}$ and illustrates the conformational diversity that branched DNA molecules may possess.

Received: July 18, 2017

Published: August 18, 2017 


\section{MATERIALS AND METHODS}

Preparation of Three-Way Junctions. Oligonucleotides were synthesized and labeled by Purimex $\mathrm{GmbH}$ (Grebenstein, Germany). The NHS esters of Alexa488 ( $5^{\prime} / 6^{\prime}$ mixed isomer, Invitrogen) or $\mathrm{Cy} 5$ (GE Healthcare) were attached via a $5-\mathrm{C}_{6}-$ aminoallyl-deoxythymidine. Annealing of samples for branched DNA was performed in buffer $[13 \mathrm{mM}$ Tris and $65 \mathrm{mM} \mathrm{NaCl}$ $(\mathrm{pH} 7.5)]$. For all structures, the ratio of donor strand to other strands was $1: 3$. Samples were heated to $90^{\circ} \mathrm{C}$ in a water bath and left to cool slowly overnight. For measurement, all samples were diluted into buffer containing $20 \mathrm{mM}$ Tris, $15 \mathrm{mM} \mathrm{NaCl}$, and $1 \mathrm{mM}$ ascorbic acid. Prior to sample addition, the buffer was stirred with activated charcoal to remove fluorescent impurities. The measurement buffer contained either 0 or 1 $\mathrm{mM} \mathrm{MgCl}$.

Sequences of Oligonucleotides for FRET Studies. The sequences used to construct the $3 \mathrm{WJ}$ s are shown below. For labeling positions, see Figure 1: 3WJa, 5' GTC GGA TCC

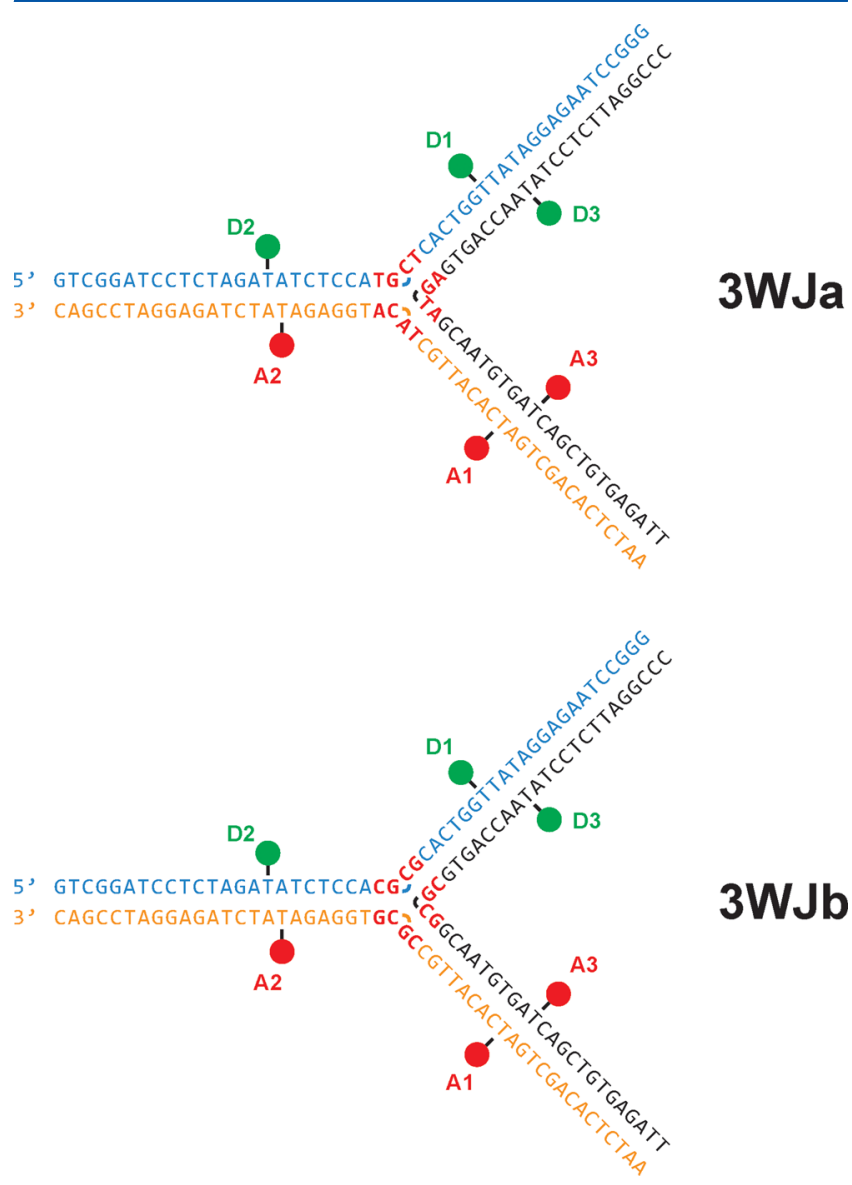

Figure 1. Sequences and dye positions for DNA three-way junctions $3 \mathrm{WJa}$ and $3 \mathrm{WJb}$ [note that this is a composite of all dye positions; measured structures have only one donor (D) and one acceptor (A) dye]. $3 \mathrm{WJa}$ and $3 \mathrm{WJb}$ differ only in the bases at the branchpoint (colored red).

TCT AGA TAT CTC CAT GCT CAC TGG TTA TAG GAG AAT CCG GG, 5' AAT CTC ACA GCT GAT CAC ATT GCT ACA TGG AGA TAT CTA GAG GAT CCG AC, 5' CCC GGA TTC TCC TAT AAC CAG TGA GTA GCA ATG TGA TCA GCT GTG AGA TT; 3WJb, 5’ GTC GGA TCC TCT AGA TAT CTC CAC GCG CAC TGG TTA TAG GAG AAT CCG GG, 5' AAT CTC ACA GCT GAT CAC ATT
GCC GCG TGG AGA TAT CTA GAG GAT CCG AC, 5' CCC GGA TTC TCC TAT AAC CAG TGC GCG GCA ATG TGA TCA GCT GTG AGA TT.

Multiparameter Fluorescence Detection (MFD). MFD is based on burstwise detection of fluorescence as single molecules diffuse through the focus of a confocal microscope. Photon counting detection by multiple detectors allows simultaneous detection of the color, lifetime, polarization, and intensity of fluorescence for each molecule. ${ }^{13}$ MFD measurements were performed using a home-built system, which we described previously. ${ }^{14}$ The process for calculating the FRET distances from the MFD data was described previously. ${ }^{5}$ The FRET distances reported here were reproducible to a $\leq 2 \AA$ standard deviation.

${ }^{19}$ F NMR Spectroscopy. Oligonucleotides were synthesized and labeled by Purimex $\mathrm{GmbH}$ and were received lyophilized; the sequences are shown below. They were initially dissolved in NMR buffer [ $20 \mathrm{mM}$ phosphate buffer ( $\mathrm{pH} 7.5$ ), $0.01 \%$ sodium azide, and $50 \mathrm{mM} \mathrm{NaCl}]$ that was prepared in ultrapure water (Direct Q3, Merck Millipore). Strands NMR_1, NMR_2, and NMR_3 were annealed together to create the $3 \mathrm{WJ}$ in a 1:2:2 ratio. NMR 1 was used alone for the single-stranded DNA (ssDNA) measurement, and NMR_1 and NMR_ 4 formed the double-stranded DNA (dsDNA) structure in a $\overline{1: 2}$ ratio. Samples were heated to $90{ }^{\circ} \mathrm{C}$ and left to cool overnight. $\mathrm{D}_{2} \mathrm{O}$ was then added to all samples to a final concentration of $5 \%$, with trimethylsilylpropionic acid (TSP) added to a final concentration of $0.00025 \%$. The $3 \mathrm{WJ}$ was also later re-lyophilized and re-dissolved in $100 \% \mathrm{D}_{2} \mathrm{O}$.

NMR data were recorded on a Bruker AVIII $500 \mathrm{MHz}$ spectrometer, fitted with a $\mathrm{BBFO}+$ probe, where the operating temperatures of the spectrometer were calibrated using a sample of D, 99.8\% methanol- $d_{4}$. The NMR spectra were processed using Topspin, and all chemical shifts are reported relative to the ${ }^{1} \mathrm{H}$ resonance of TSP, using the position of the TSP signal in the corresponding ${ }^{1} \mathrm{H}$ one-dimensional (1D) spectra, recorded across the temperature range for reference. ${ }^{15}$

Sequences of Oligonucleotides for NMR Studies. The sequences for the $3 \mathrm{WJ}$, ssDNA, and dsDNA that were studied using ${ }^{19} \mathrm{~F}$ NMR spectroscopy are shown below. The position of the 5-fluorocytosine is underlined: NMR_1, 5' TAT CTC CAC GCG CAC TGG TT; NMR 2, 5' AAC CAG TGC GCG GCA ATG TG; NMR_3, 5' CAC ATT GCC GCG TGG AGA TA; NMR 4, 5' AAC CAG TGC GCG TGG AGA TA.

Ensemble Optical Spectroscopy. Absorption spectra were recorded on a Cary 50 (Agilent Technologies) spectrometer. Steady-state and time-resolved fluorescence were measured under magic angle conditions using a fluorescence spectrometer (FluoTime 300, Picoquant GmbH) with a hybrid PMT detector (PMA Hybrid 40, Picoquant). The absorbance of the sample was low $(<0.05)$, so that inner-filter effects were negligible. Time-resolved fluorescence spectroscopy was performed using the technique of time-correlated singlephoton counting. The excitation source was a super continuum laser (WL-SC-400-4-PP, Fianium) with a tunable wavelength filter and a multimode fiber (Superchrome-Vis-FDS-MM, Fianium) at a wavelength of $488 \mathrm{~nm}$, a bandpass of $10 \mathrm{~nm}$, and a repetition rate of $10 \mathrm{MHz}$. The instrument response function (IRF) was $\sim 130$ ps full width at half-maximum; the time per channel was 8 ps. Decay curves were analyzed using a standard iterative reconvolution method (FluoFit, Picoquant $\mathrm{GmbH}$ ), assuming a multiexponential decay function. The 


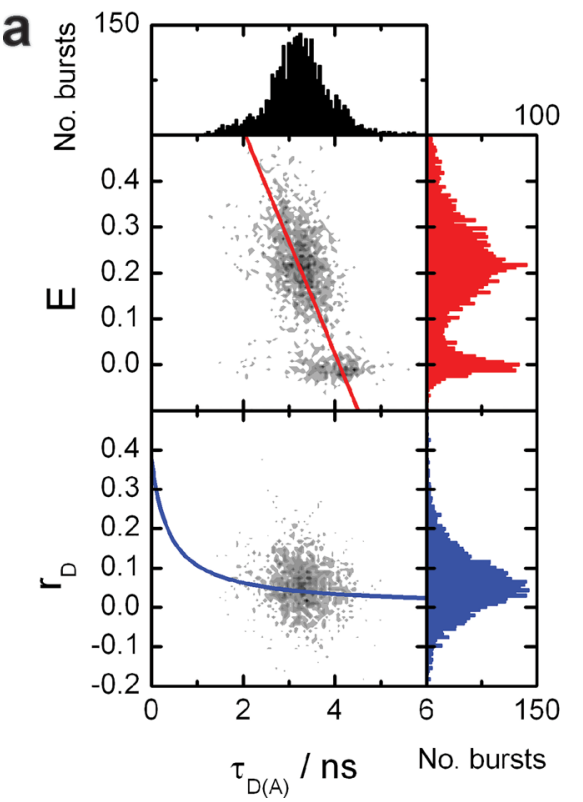

b

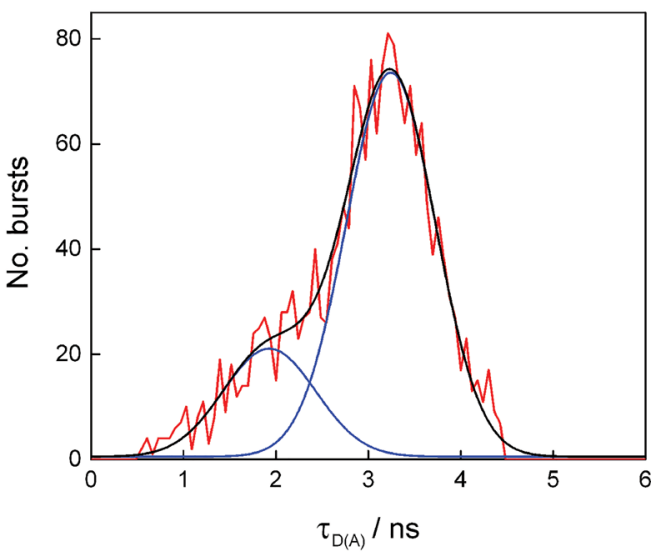

Figure 2. Single-molecule FRET shows a GC-rich 3WJ adopts multiple conformations. (a) Two-dimensional burst frequency histograms of FRET efficiency $(E)$ or donor anisotropy $\left(r_{\mathrm{D}}\right)$ vs donor lifetime $\left[\tau_{\mathrm{D}(\mathrm{A})}\right]$. The overlaid red line is the theoretical FRET relationship $E=1-\left[\tau_{\mathrm{D}(\mathrm{A})} / \tau_{\mathrm{D}}\right]$, where $\tau_{\mathrm{D}}=4.1 \mathrm{~ns}$. The blue overlaid line is the Perrin equation $r_{\mathrm{D}}=r_{0} /\left[1+\tau_{\mathrm{D}(\mathrm{A})} / \rho_{\mathrm{D}}\right]$, where mean rotational correlation time $\rho_{\mathrm{D}}=0.35 \mathrm{~ns}$ and fundamental anisotropy $r_{0}=0.375$. (b) A sub-ensemble analysis of the whole FRET population can be fitted (black) as two Gaussian distributions (blue). The data in panels a and $\mathrm{b}$ are for $3 \mathrm{WJb}$ in $0 \mathrm{mM} \mathrm{MgCl}_{2}$ (dye positions D1 and A1) but were recorded on different days.

quality of the fit was judged on the basis of the reduced $\chi^{2}$ statistic and the randomness of residuals.

Molecular Dynamics (MD) Simulations. The procedure for deriving structures from FRET distance restraints was described previously for $3 \mathrm{WJa} .{ }^{5}$

For the starting model, the three DNA arms of the three-way junction were modeled as B-DNA using the program Hyperchem (version 8.0.8 for Windows, Molecular Modeling System, Lightwave Scientific). The program $\mathrm{CNS}^{16}$ was then used for the simulated annealing molecular dynamics calculation. During the simulation, FRET distance restraints were applied to model the geometry of the DNA. We used the mean dye positions for Alexa488 and Cy5 obtained from a MD simulation that had been published previously. ${ }^{17}$

In brief, the mean positions were fixed relative to the surface of the DNA by distance restraints. The distance restraints were generated by the DEN (Deformable Elastic Network) feature ${ }^{18}$ in CNS that selects random atom pairs that are within a specified distance range in the starting structure. It should be noted that we did not actually use deformable restraints but rather used regular (nondeformable) harmonic distance restraints during the refinement.

\section{RESULTS}

GC-Rich 3WJ Structure from Single-Molecule FRET and MD. To test whether the unpairing of bases at the branchpoint of fully complementary $3 \mathrm{WJs}$ is a general phenomenon or is specific to the previously studied $3 \mathrm{WJ}$ (here named $3 \mathrm{WJa}$ ), we modified the sequence so that the four nucleotides next to the branchpoint in each arm had the potential to form two G:C Watson-Crick pairs (3WJb). The sequences of $3 \mathrm{WJa}$ and $3 \mathrm{WJb}$ are shown in Figure 1, together with the positions used for fluorescent dye labeling; the 12 nucleotides closest to the branchpoint are colored red. We postulated that the GC-rich branchpoint of $3 \mathrm{WJb}$ might make the formation of a fully paired structure more likely.

We studied 3WJs labeled with one donor dye and one acceptor dye. The six dye positions (Figure 1) allowed seven different combinations of donor and acceptor dyes to be used for single-molecule FRET measurements using MFD. ${ }^{13}$ MFD is ideal for measuring accurate dye-dye distances for structural determination. ${ }^{19}$ For each FRET pair, $3 \mathrm{WJb}$ was measured in a buffer containing 0 or $1 \mathrm{mM} \mathrm{Mg}^{2+}$, to check for potential ioninduced effects. In each case, the data were qualitatively similar to those for $3 \mathrm{WJa}$, showing a donor-only peak and a FRET population (Figure $2 \mathrm{a}$ ). MFD data for all the $3 \mathrm{WJb}$ samples are given in Figures $S 1$ and $S 2$.

Although the MFD plots for 3WJa and 3WJb are qualitatively similar, upon closer inspection of the MFD plots it is apparent that the FRET populations for 3WJb (Figures 2a, S1, and S2) are much broader and less symmetric than those of $3 \mathrm{WJa}$ and other branched DNA structures that we have examined previously. ${ }^{5,14}$ The three broadest FRET distributions (for the D1-A1, D1-A3, and D3-A1 pairs) could be reliably fitted to double Gaussian distributions (see Table S1). For example, the FRET lifetime distribution in Figure 2a can be fitted with two Gaussians (Figure $2 \mathrm{~b}$ ) with lifetimes of $1.93(0.1) \mathrm{ns}(23 \%)$ and $3.24(0.03) \mathrm{ns}(77 \%)$. In these cases, the minor population, which ranged from 17 to $34 \%$, has a higher FRET efficiency. In contrast, for the other FRET pairs, the distribution of lifetimes could not be reliably separated and the distribution fits a single Gaussian. These results can be explained by the existence of a minor population, which adopts a conformation in which two arms are closer to each other than in the major species. The D1-A1, D1-A3, and D3-A1 FRET pairs are all associated with the same two arms, which might mean that FRET distances involving the third arm are less affected by a change in conformation. Notably, the entire FRET population lies along the theoretical FRET line, implying that there are at least two conformations that are static or in slow exchange on the 
millisecond time scale required for diffusion through the confocal volume. ${ }^{20}$ A direct comparison between the MFD plots of $3 \mathrm{WJa}$ and $3 \mathrm{WJb}$ for these three FRET pairs illustrates the very different shapes and breadths of their FRET distributions in 0 and $1 \mathrm{mM} \mathrm{Mg}^{2+}$ (Figures S3 and S4, respectively). In support of the SM-FRET data, bulk lifetime measurements of $3 \mathrm{WJb}$ (dye position $\mathrm{D} 1$ and $\mathrm{A} 1$ in $1 \mathrm{mM}$ $\mathrm{Mg}^{2+}$ ) using time-correlated single-photon counting (TCSPC) were clearly biexponential (Figure S5), with lifetimes and weights similar to those of the SM-FRET sub-ensemble fits.

To derive a structure from the SM-FRET data for $3 \mathrm{WJb}$ comparable to that calculated for $3 \mathrm{WJa},{ }^{5}$ we fitted a small region of the peak of the MFD distribution (the region had a width in $\tau$ of 0.7 ns and a height in $E$ of 0.07 ); importantly, the lifetime recovered from a single Gaussian fit of this small region matched the lifetime of the major population measured by a double Gaussian fit to the whole FRET region (see Table S1). The fluorescence lifetime, $\tau_{\mathrm{D}(\mathrm{A})}$, for the major FRET population was extracted from three separate measurements and converted to a dye-dye distance. The average $\tau_{\mathrm{D}(\mathrm{A})}$ from these experiments and the resulting dye-dye distances $(r)$ are listed in Table 1 . The global structure was modeled using molecular

Table 1. FRET Species Lifetimes $\left[\tau_{\mathrm{D}(\mathrm{A})}\right]$ and Calculated Dye-Dye Distances $(r)$ for the Major $3 \mathrm{WJb}$ Structure $^{a}$

\begin{tabular}{ccccccccc} 
& \multicolumn{3}{c}{$0 \mathrm{mM} \mathrm{Mg}^{2+}$} & & \multicolumn{3}{c}{$1 \mathrm{mM} \mathrm{Mg}^{2+}$} \\
\cline { 2 - 3 } \cline { 7 - 8 } structure & $\begin{array}{c}\tau_{\mathrm{D}(\mathrm{A})} \\
(\mathrm{ns})\end{array}$ & $r(\AA)$ & $\begin{array}{c}\text { 3WJa } r \\
(\AA)\end{array}$ & & $\begin{array}{c}\tau_{\mathrm{D}(\mathrm{A})} \\
(\mathrm{ns})\end{array}$ & $r(\AA)$ & $\begin{array}{c}\text { 3WJa } r \\
(\AA)\end{array}$ \\
$\mathrm{D} 1-\mathrm{A} 1$ & 3.20 & $64(0.9)$ & 69 & & 3.10 & $63(<0.1)$ & 66 \\
$\mathrm{D} 1-\mathrm{A} 2$ & 3.15 & $63(0.2)$ & 64 & & 2.99 & $61(<0.1)$ & 62 \\
$\mathrm{D} 1-\mathrm{A} 3$ & 3.13 & $63(0.9)$ & 68 & & 3.09 & $62(0.6)$ & 67 \\
$\mathrm{D} 2-\mathrm{A} 1$ & 3.41 & $69(0.7)$ & 71 & & 3.19 & $64(0.4)$ & 65 \\
$\mathrm{D} 2-\mathrm{A} 3$ & 3.69 & $76(2.3)$ & 81 & & 3.49 & $70(1.7)$ & 78 \\
$\mathrm{D} 3-\mathrm{A} 1$ & 3.25 & $65(1.5)$ & 69 & & 3.18 & $64(0.7)$ & 67 \\
$\mathrm{D} 3-\mathrm{A} 2$ & 3.28 & $66(0.3)$ & 66 & & 3.07 & $62(0.1)$ & 64
\end{tabular}

${ }^{a}$ Distances for $3 \mathrm{WJa}$ are also shown for comparison. Lifetimes were recovered from a single Gaussian fit of a sub-ensemble region centered on the FRET maximum of MFD data (the selected region had a width in $\tau$ of $0.7 \mathrm{~ns}$ and a height in $E$ of 0.07 ). The standard deviation for $r$ is indicated in parentheses.

dynamics simulations (MD) as done previously for $3 \mathrm{WJa},{ }^{5}$ treating the arms as duplexes with B-form structure, while using the FRET distances listed in Table 1 as structural restraints. The FRET distances were imposed as the distance $\pm 0.5 \AA$ using a harmonic square well potential. Four different sets of restraints were applied, differing in the number of bases paired at the junction (termed 0 -free, 1 -free, 2 -free, and 3 -free). The distance root-mean-square deviations (DRMSDs) between the model generated and the experimental dye-dye distances are listed in Table S2; these DRMSDs are a measure of how well the structures fit the FRET restraints. As with $3 \mathrm{WJa}$, the model with the lowest DRMSD, and therefore the model that most closely matches the experimentally measured FRET distances, is the 2-free model. In other words, the FRET restraints produce a structure with unpairing at the branchpoint. The five lowest-restraint energy conformations with the 2 -free restraint applied are shown in Figure 3.

As seen for $3 \mathrm{WJa},{ }^{5}$ there is little change in structure upon addition of $1 \mathrm{mM} \mathrm{Mg}^{2+}$ (Figure 3a). Comparison of the $3 \mathrm{WJa}$ ensemble and the major conformation of $3 \mathrm{WJb}$ (Figure $3 \mathrm{~b}$ and Figure S6) shows that despite the significant differences in the
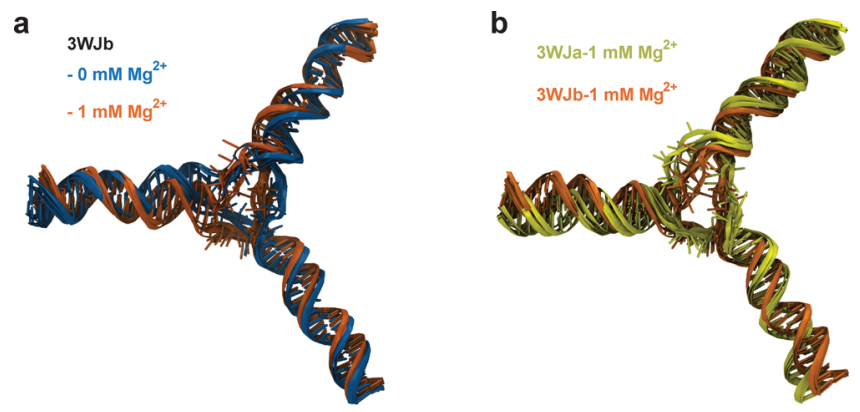

Figure 3. Global structure of 3WJb derived from SM-FRET distance restraints for the major FRET population and MD simulations showing that the unpaired branchpoint is present with and without $\mathrm{MgCl}_{2}$ (a) and that the structures for $3 \mathrm{WJa}$ (yellow) and the major conformation of $3 \mathrm{WJb}$ (orange) are similar (b). The five lowestrestraint energy conformations are superposed using the DNA backbone atoms.

FRET distances measured between some donor-acceptor pairs (Table 1), 3WJb clearly adopts an extended Y-shaped conformation similar to that of $3 \mathrm{WJa}$ (Figure $3 \mathrm{~b}$ ) with no evidence of coaxial stacking of the arms at either 0 or $1 \mathrm{mM}$ $\mathrm{Mg}^{2+}$

GC-Rich 3WJ Conformation Monitored by ${ }^{19} \mathrm{~F}$ NMR Spectroscopy. SM-FRET and TCSPC measurements provided strong evidence that $3 \mathrm{WJb}$ can adopt at least two distinct conformations. To corroborate the findings from the fluorescence measurements and characterize the conformations of the bases at the junction site, we sought to apply solution NMR spectroscopy. However, while ${ }^{1} \mathrm{H}$ NMR structures of $3 \mathrm{WJ}$ s that incorporate bulges in one strand have been determined, ${ }^{21,22}$ previous attempts to characterize perfectly complementary $3 \mathrm{WJ}$ s reportedly failed. ${ }^{23}$ The most likely explanation for this is that the particular dynamic nature of the systems studied resulted in the critical ${ }^{1} \mathrm{H}$ signals being broadened to an extent that they were either undetectable or could not be resolved, likely because of intermediate exchange.

Because the effects of such exchange broadening depend on the difference in frequency between the NMR signals of the exchanging species, we reasoned that the synthesis of oligonucleotides that incorporated an NMR active nucleus with a frequency range much wider than that of ${ }^{1} \mathrm{H}$ could restore the ability to observe signals in the slow exchange regime. ${ }^{19} \mathrm{~F}$ has a chemical shift range much wider than that of ${ }^{1} \mathrm{H}$, is highly sensitive to its chemical environment, and can be stably incorporated into nucleotide analogues, allowing the site specific labeling of synthetic oligonucleotides. ${ }^{24,25}$ Fluorine's chemical shift range is wider than that of ${ }^{1} \mathrm{H}$, and because its gyromagnetic ratio is similar, its NMR signals are spread over a much wider range of frequencies at a particular magnetic field strength. We therefore designed a $3 \mathrm{WJ}$ made from three 20 mers of identical sequence to the central 20 nucleotides used in $3 \mathrm{WJb}$ and labeled at the branchpoint with 5-fluoro-dC (Figure 4a). Although the ${ }^{19} \mathrm{~F} 3 \mathrm{WJ}$ is a truncated version of $3 \mathrm{WJb}$, it should still be very stable, as there should be at least 8 bp in each arm.

We also recorded data from samples of the ${ }^{19} \mathrm{~F}$-labeled oligonucleotide alone and incorporated into a fully complementary duplex structure, to establish reference chemical shifts for single- and double-stranded conformations. In $1 \mathrm{D}{ }^{19} \mathrm{~F}$ spectra measured at $470 \mathrm{MHz}\left(11.4 \mathrm{~T}, 500 \mathrm{MHz}\right.$ for $\left.{ }^{1} \mathrm{H}\right)$, our single-stranded $20 \mathrm{mer}$ with a $5 \mathrm{~F}-\mathrm{dC}$ at position 11 contains two 


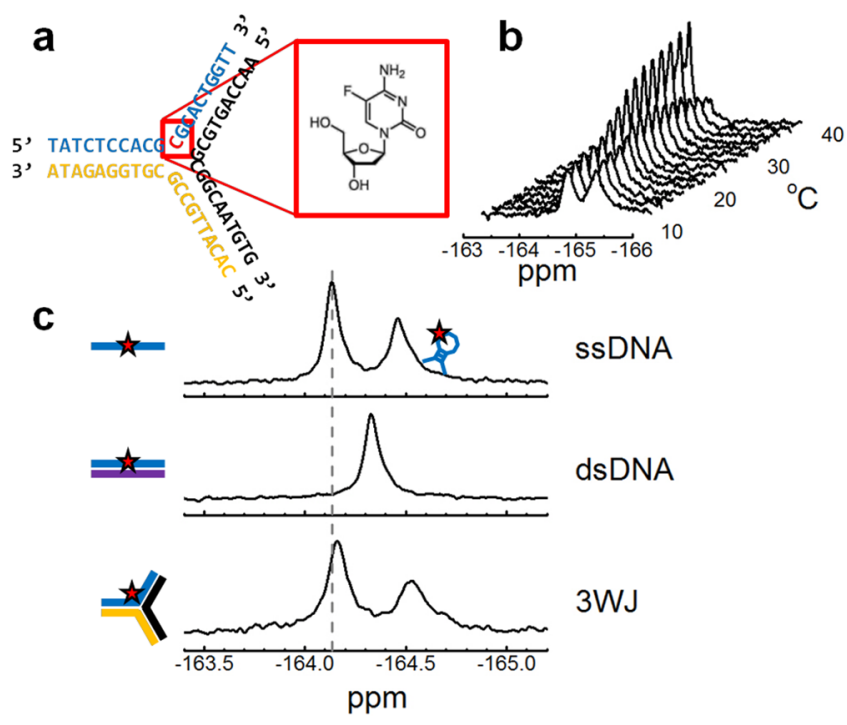

Figure 4. NMR spectra of a ${ }^{19} \mathrm{~F}$-labeled $3 \mathrm{WJ}$. A $3 \mathrm{WJ}$ with a GC-rich branchpoint was modified to include a 5-fluorodeoxycytosine. (a) The schematic shows the sequence of the $3 \mathrm{WJ}$, where the position of the $5 \mathrm{~F}-\mathrm{dC}$ is colored red, and its chemical structure is given in the inset. (b) $1 \mathrm{D}{ }^{19} \mathrm{~F}$ NMR spectra of the $3 \mathrm{WJ}$ at temperatures from 12 to 40 ${ }^{\circ} \mathrm{C}$. The $3 \mathrm{WJ}$ spectra show two major peaks that become broader with a decrease in temperature. (c) $1 \mathrm{D}^{19} \mathrm{~F}$ NMR spectra of ssDNA (top), dsDNA (middle), and the $3 \mathrm{WJ}$ (bottom) at $30{ }^{\circ} \mathrm{C}$. Note that the ssDNA is capable of forming a hairpin, positioning the $5 \mathrm{~F}-\mathrm{dC}$ in a loop environment that results in the more upfield peak. The more downfield peak represents a ssDNA conformation that has no defined secondary structure. The vertical dashed line marks the position of the ssDNA peak.

peaks at $30{ }^{\circ} \mathrm{C}$, indicating that it acts as a bistable oligonucleotide exchanging between a single-stranded conformation and a second conformation that is most likely a $4 \mathrm{bp}$ double-stranded hairpin structure in which the $5 \mathrm{~F}-\mathrm{dC}$ is found at the $5^{\prime}$ position of the intervening four-nucleotide loop (Figure 4c). We can assign the downfield peak to the singlestranded conformation because it becomes more highly populated with an increase in temperature (Figure S5b). In contrast, in the double-stranded state, the $5 \mathrm{~F}$-dC gives rise to the expected single, sharp peak at a chemical shift between the single-stranded and loop chemical shifts (Figure 4c), whose position changes little at temperatures from 12 to $40{ }^{\circ} \mathrm{C}$ (Figure S7).

A $5 \mathrm{~F}-\mathrm{dC}$ label has previously been used to study DNA secondary structure. ${ }^{25}$ This study found that the thermodynamic stability of labeled and unlabeled DNA and RNA molecules was unaffected by labeling. The chemical shift of the ${ }^{19} \mathrm{~F}$ can be influenced by a number of factors, including the identity of the bases stacked on either side of the GC base pair, whether the labeled base is at the end of the dsDNA, and whether the nucleic acid is an A- or B-form helix. Similarly, noncanonical structures could result in different magnetic environments. Although the dsDNA chemical shift we observe is not as upfield as previously reported (by $\sim 0.5 \mathrm{ppm}$ ), this is likely because differences in sequence context exist (ACXTT in ref 25 compared to CGXGC) and also because our spectra were recorded in $95 \% \mathrm{H}_{2} \mathrm{O}$ (the $5-{ }^{19} \mathrm{~F}$-dC resonance shifts progressively downfield as $\mathrm{H}_{2} \mathrm{O}$ is added to a $\mathrm{D}_{2} \mathrm{O}$ solution, which is attributed to a secondary isotope effect caused by nearby imino hydrogens ${ }^{25}$ ).
The $1 \mathrm{D}{ }^{19} \mathrm{~F}$ spectrum of the $5 \mathrm{~F}-\mathrm{dC}$ in the assembled $3 \mathrm{WJ}$ is characterized by the presence of two distinct peaks at -164.16 and $-164.52 \mathrm{ppm}$ indicating that the $5 \mathrm{~F}-\mathrm{dC}$ is sampling (at least) two distinct conformations (Figure $4 \mathrm{~b}$ ). The relative intensities of these peaks change with temperature, indicating that the states are in exchange. The downfield peak's chemical shift is very close to the single-stranded chemical shift, which we interpret to mean that the base spends a significant proportion of the time in an unstacked, single-stranded conformation. The small chemical shift difference with ssDNA could be due to the exchange process. Additionally, in unstructured ssDNA, the chemical shift is a product of rapid averaging among many different environments. If the constrained $3 \mathrm{WJ}$ context changes the weighting of the populations sampled, that would also change the shift. The upfield peak matches neither the double-stranded chemical shift nor the loop shift seen for the bistable oligonucleotide and is further upfield than either (Figure 4c). There are a number of possible conformations that could give rise to such an upfield shift, including non-B-form double-stranded structures or placement in the last base pair of a double-stranded region. The similar pattern of peaks seen for the $3 \mathrm{WJ}$ in $100 \% \mathrm{D}_{2} \mathrm{O}$ (Figure S5) excludes the possibility that the spectral features seen in $\mathrm{H}_{2} \mathrm{O}$ arise from secondary isotope effects.

Fitting the $3 \mathrm{WJ}$ spectrum at $30^{\circ} \mathrm{C}$ with Dynamic $\mathrm{NMR}^{26,27}$ (TEDDY DNMR) suggests that the true chemical shifts of the exchanging species are very close to the shifts observed and that the rate of exchange between the states is around $15 \mathrm{~Hz}$ with a $60 / 40$ population ratio. This agrees with our interpretation of the SM-FRET data as representing two or more conformations that are stable on the time scale of diffusion through the confocal volume (several milliseconds).

\section{DISCUSSION}

Pioneering ensemble studies had previously illustrated the power of FRET to probe the conformation and heterogeneity of branched DNA molecules such as $3 \mathrm{WJ} s^{28,29}$ The emergence of SM-FRET as a powerful approach to determining structure $^{17,19,30-32}$ has subsequently made it possible to unambiguously probe the structure and heterogeneity of branched DNA in unprecedented detail. ${ }^{5,14}$ In an earlier report, SM-FRET and MD were combined with ensemble timeresolved fluorescence of a nucleobase analogue (2-AP) to yield information about both global structure (Y-shaped with pyramidal geometry) and local structure (observation of an unpaired branchpoint) in a fully complementary $3 \mathrm{WJ} .{ }^{5}$ Although this work reconciled much of the previous literature about this class of branched molecules, it was not known whether this was a general result for all fully complementary 3WJs.

In this work, we have attempted to address this issue by studying a $3 \mathrm{WJ}$ with a GC-rich branchpoint, hypothesizing that this could provide the stability required for full Watson-Crick pairing. Although the changes to the overall sequence were small, amounting to only four AT pairs exchanged for GCs, the change was clearly manifested in the heterogeneous SM-FRET data, which was interpreted as arising from at least two different conformations. The combination of FRET restraints from the major conformation of $3 \mathrm{WJb}$ and $\mathrm{MD}$ produced a global structure that was in excellent agreement with that derived for $3 \mathrm{WJa}$, with an asymmetric $\mathrm{Y}$ shape and a shallow pyramidal geometry. This is also in agreement with recent small angle $\mathrm{X}$ ray scattering (SAXS) experiments on a $3 \mathrm{WJ}$, which confirmed 
that the arms are in a B form and that the global structure is asymmetric. $^{33}$

The lack of an adenine at the branchpoint precluded us from utilizing 2-AP as a probe of local branch structure, as was done for $3 \mathrm{WJa} .{ }^{5}$ Instead, we used ${ }^{19} \mathrm{~F}$ to label a cytosine at the branch, thereby allowing us to report the first NMR spectra of a branchpoint base on a fully complementary $3 \mathrm{WJ}$. The two resonances observed for the $3 \mathrm{WJ}$ can be assigned as the cytosine in different environments: one resonance resembles that of the same nucleotide in ssDNA, while the other indicates that the base populates a distinct DNA secondary structure. The latter conformation does not match either dsDNA or the hairpin structure formed for the labeled single strand; therefore, it may be due to a distorted Watson-Crick pair, an underwound helical conformation, or alternative secondary structure.

We did not attempt to model the minor conformation(s) observed via SM-FRET as we did for the major species. Even if we assume that there are only two conformations, we were able to reliably assign different FRET populations, and therefore derive distances, for only the most heterogeneous FRET distributions. The simplest model that is consistent with the SM-FRET and NMR data is for a $3 \mathrm{WJ}$ that can adopt two conformations, which interconvert on a time scale of tens of milliseconds (Figure 5). However, we note that we cannot rule out the possibility of multiple secondary conformations.

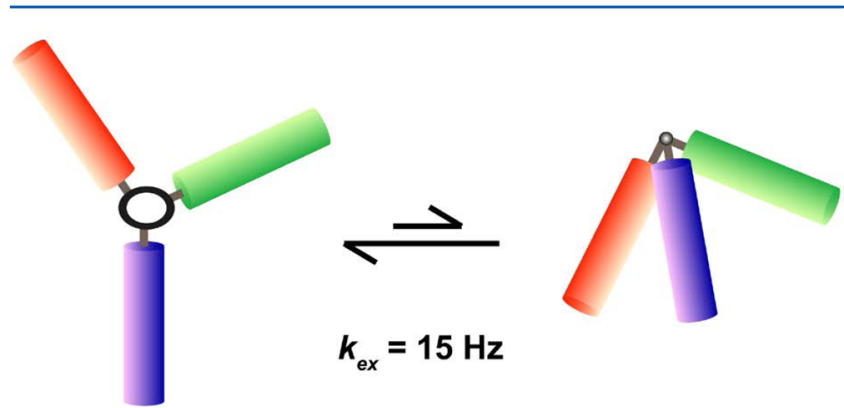

Figure 5. Simple model of $3 \mathrm{WJ}$ conformational heterogeneity assuming a two-state system. The left image represents the major open structure derived from FRET restraints (Figure 3), which can convert via base pairing at the branch to a constrained structure in which there are strengthened interactions at the branchpoint and two of the arms are closer together. The exchange rate is an estimate from DNMR measurements (see the text).

This work demonstrates that SM-FRET, MD, and NMR spectroscopy are a powerful combination of techniques for studying nucleic acid structures. They have been used here to demonstrate the structural complexity in a simple branched nucleic acid molecule, a DNA 3WJ. The observed conformational heterogeneity may have an impact on the accessibility and reactivity of the $3 \mathrm{WJ}$ branchpoint, and the manner in which they are recognized and processed by enzymes. It also indicates the potential for subtle control of DNA structure and dynamics at the nanoscale through judicious choice of sequence and environment.

\section{ASSOCIATED CONTENT}

\section{S Supporting Information}

The Supporting Information is available free of charge on the ACS Publications website at DOI: 10.1021/acs.biochem.7b00677.
Single-molecule FRET plots for $3 \mathrm{WJa}$ and $3 \mathrm{WJb}$; TCSPC data for $3 \mathrm{WJb}$; overlaid molecular models of the global structure of $3 \mathrm{WJa}$ and $3 \mathrm{WJb}$ in $0 \mathrm{mM} \mathrm{Mg}^{2+}$ buffer; 1D ${ }^{19} \mathrm{~F}$ NMR spectra of a GC-rich $3 \mathrm{WJ}$ in $\mathrm{D}_{2} \mathrm{O}$ from 12 to $40{ }^{\circ} \mathrm{C}$, ssDNA in $\mathrm{H}_{2} \mathrm{O}$ from 21 to $30^{\circ} \mathrm{C}$, and dsDNA in $\mathrm{H}_{2} \mathrm{O}$ from 12 to $38^{\circ} \mathrm{C}$; sub-ensemble analysis of $3 \mathrm{WJb}$ FRET data; and DRMSD from the FRET-based restraints in $3 \mathrm{WJb}(\mathrm{PDF})$

\section{AUTHOR INFORMATION}

\section{Corresponding Author}

*E-mail: steven.magennis@glasgow.ac.uk.

\section{ORCID}

Laura E. Baltierra-Jasso: 0000-0002-4824-5926

Steven W. Magennis: 0000-0002-4327-8366

\section{Present Address}

$\nabla_{\text {T.S.: }}$ School of Clinical and Applied Sciences, Leeds Beckett University, Portland Building, City Campus, Leeds LS1 3HE, U.K.

\section{Funding}

This work was supported by EPSRC Grant EP/L027003/1 (M.J.M.), BBSRC Grant BB/G00269X/1 (T.S.), and a CONACyT scholarship (L.E.B.-J.).

\section{Notes}

The authors declare no competing financial interest.

\section{REFERENCES}

(1) Lilley, D. M. J. (2000) Structures of helical junctions in nucleic acids. Q. Rev. Biophys. 33, 109-159.

(2) Atkinson, J., and McGlynn, P. (2009) Replication fork reversal and the maintenance of genome stability. Nucleic Acids Res. 37, 34753492.

(3) Seeman, N. C. (2010) Nanomaterials based on DNA. Annu. Rev. Biochem. 79, 65-87.

(4) Roy, R., Hohng, S., and Ha, T. (2008) A practical guide to singlemolecule FRET. Nat. Methods 5, 507-516.

(5) Sabir, T., Toulmin, A., Ma, L., Jones, A. C., McGlynn, P., Schroeder, G. F., and Magennis, S. W. (2012) Branchpoint Expansion in a Fully Complementary Three-Way DNA Junction. J. Am. Chem. Soc. 134, 6280-6285.

(6) Young, R. M., Singh, A. P. N., Thazhathveetil, A. K., Cho, V. Y., Zhang, Y. Q., Renaud, N., Grozema, F. C., Beratan, D. N., Ratner, M. A., Schatz, G. C., Berlin, Y. A., Lewis, F. D., and Wasielewski, M. R. (2015) Charge Transport across DNA-Based Three-Way Junctions. J. Am. Chem. Soc. 137, 5113-5122.

(7) Zhang, Y. Q., Young, R. M., Thazhathveetil, A. K., Singh, A. P. N., Liu, C. R., Berlin, Y. A., Grozema, F. C., Lewis, F. D., Ratner, M. A., Renaud, N., Siriwong, K., Voityuk, A. A., Wasielewski, M. R., and Beratan, D. N. (2015) Conformationally Gated Charge Transfer in DNA Three-Way Junctions. J. Phys. Chem. Lett. 6, 2434-2438.

(8) Zhang, Y. Q., Zhang, W. B., Liu, C. R., Zhang, P., Balaeff, A., and Beratan, D. N. (2016) DNA charge transport: Moving beyond 1D. Surf. Sci. 652, 33-38.

(9) Ma, L., Wu, G. R., Li, Y. F., Qin, P., Meng, L. P., Liu, H. Y., Li, Y. Y., and Diao, A. P. (2015) A reversible metal ion fueled DNA threeway junction molecular device for "turn-on and -off" fluorescence detection of mercury ions (II) and biothiols respectively with high selectivity and sensitivity. Nanoscale 7, 18044-18048.

(10) Barros, S. A., and Chenoweth, D. M. (2014) Recognition of Nucleic Acid Junctions Using Triptycene-Based Molecules. Angew. Chem., Int. Ed. 53, 13746-13750.

(11) Probst, M., Langenegger, S. M., and Haner, R. (2014) A modular LHC built on the DNA three-way junction. Chem. Commun. 50, 159-161. 
(12) Su, W., Bagshaw, C. R., and Burley, G. A. (2013) Addressable and unidirectional energy transfer along a DNA three-way junction programmed by pyrrole-imidazole polyamides. Sci. Rep. 3, 1883.

(13) Kühnemuth, R., and Seidel, C. A. M. (2001) Principles of single molecule multiparameter fluorescence spectroscopy. Single Mol. 2, 251-254.

(14) Sabir, T., Schröder, G. F., Toulmin, A., McGlynn, P., and Magennis, S. W. (2011) Global structure of forked DNA in solution revealed by high-resolution single-molecule FRET. J. Am. Chem. Soc. 133, 1188-1191.

(15) Harris, R. K., Becker, E. D., Cabral De Menezes, S. M., Goodfellow, R., and Granger, P. (2001) NMR nomenclature. Nuclear spin properties and conventions for chemical shifts - (IUPAC recommendations 2001). Pure Appl. Chem. 73, 1795-1818.

(16) Brunger, A. T. (2007) Version 1.2 of the Crystallography and NMR system. Nat. Protoc. 2, 2728-2733.

(17) Woźniak, A. K., Schröder, G. F., Grubmüller, H., Seidel, C. A M., and Oesterhelt, F. (2008) Single-molecule FRET measures bends and kinks in DNA. Proc. Natl. Acad. Sci. U. S. A. 105, 18337-18342.

(18) Schröder, G. F., Brunger, A. T., and Levitt, M. (2007) Combining efficient conformational sampling with a deformable elastic network model facilitates structure refinement at low resolution. Structure 15, 1630-1641.

(19) Kalinin, S., Peulen, T., Sindbert, S., Rothwell, P. J., Berger, S., Restle, T., Goody, R. S., Gohlke, H., and Seidel, C. A. M. (2012) A toolkit and benchmark study for FRET-restrained high-precision structural modeling. Nat. Methods 9, 1218-1225.

(20) Kalinin, S., Valeri, A., Antonik, M., Felekyan, S., and Seidel, C. A. M. (2010) Detection of structural dynamics by FRET: a photon distribution and fluorescence lifetime analysis of systems with multiple states. J. Phys. Chem. B 114, 7983-7995.

(21) Leontis, N. B., Hills, M. T., Piotto, M., Malhotra, A., Nussbaum, J., and Gorenstein, D. G. (1993) A model for the solution structure of a branched, 3-strand DNA complex. J. Biomol. Struct. Dyn. 11, 215223.

(22) Wu, B., Girard, F., van Buuren, B., Schleucher, J., Tessari, M., and Wijmenga, S. (2004) Global structure of a DNA three-way junction by solution NMR: towards prediction of $3 \mathrm{H}$ fold. Nucleic Acids Res. 32, 3228-3239.

(23) Ouporov, I. V., and Leontis, N. B. (1995) Refinement of the solution structure of a branched DNA 3-way junction. Biophys. J. 68, 266-274.

(24) Seela, F., and Xu, K. (2008) DNA with stable fluorinated dA and dG substitutes: syntheses, base pairing and F-19-NMR spectra of 7fluoro-7-deaza-2 '-deoxyadenosine and 7-fluoro-7-deaza-2 '-deoxyguanosine. Org. Biomol. Chem. 6, 3552-3560.

(25) Puffer, B., Kreutz, C., Rieder, U., Ebert, M.-O., Konrat, R., and Micura, R. (2009) 5-Fluoro pyrimidines: labels to probe DNA and RNA secondary structures by 1D F-19 NMR spectroscopy. Nucleic Acids Res. 37, 7728-7740.

(26) Rohonczy, J. (1992) Total lineshape analysis of DNMR spectra by IBM personal computer. Kem. Kozl. 74, 161-200.

(27) Rohonczy, J. (2007) DNMR Lineshape Analysis Software Manual, version 1.1, revision 071103, Bruker BioSpin GmbH, Rheinstetten, Germany.

(28) Yang, M. S., and Millar, D. P. (1996) Conformational flexibility of three-way DNA junctions containing unpaired nucleotides. Biochemistry 35, 7959-7967.

(29) Stühmeier, F., Welch, J. B., Murchie, A. I. H., Lilley, D. M. J., and Clegg, R. M. (1997) Global structure of three-way DNA junctions with and without additional unpaired bases: A fluorescence resonance energy transfer analysis. Biochemistry 36, 13530-13538.

(30) Sisamakis, E., Valeri, A., Kalinin, S., Rothwell, P. J., and Seidel, C. A. M. (2010) Accurate single-molecule FRET studies using multiparameter fluorescence detection. Methods Enzymol. 475, 455514.

(31) Muschielok, A., Andrecka, J., Jawhari, A., Brueckner, F., Cramer, P., and Michaelis, J. (2008) A nano-positioning system for macromolecular structural analysis. Nat. Methods 5, 965-971.
(32) Brunger, A. T., Strop, P., Vrljic, M., Chu, S., and Weninger, K. R. (2011) Three-dimensional molecular modeling with single molecule FRET. J. Struct. Biol. 173, 497-505.

(33) Im, K., Jeong, D., Hur, J., Kim, S. J., Hwang, S., Jin, K. S., Park, N., and Kim, K. (2013) Robust analysis of synthetic label-free DNA junctions in solution by X-ray scattering and molecular simulation. Sci. Rep. 3, 3226. 